\title{
Randomised placebo controlled multicentre trial to assess short term clarithromycin for patients with stable coronary heart disease: CLARICOR trial
}

Christian M Jespersen, Bodil Als-Nielsen, Morten Damgaard, Jørgen Fischer Hansen, Stig Hansen, Olav H Helø, Per Hildebrandt, Jørgen Hilden, Gorm Boje Jensen, Jens Kastrup, Hans Jørn Kolmos, Erik Kjøller, Inga Lind, Henrik Nielsen, Lars Petersen, Christian Gluud, the CLARICOR Trial Group

\begin{abstract}
Objective To determine if the macrolide clarithromycin affects mortality and cardiovascular morbidity in patients with stable coronary heart disease.

Design Centrally randomised multicentre trial. All parties at all stages were blinded. Analyses were by intention to treat.

Setting Five Copenhagen University cardiology departments and a coordinating centre.

Participants 13702 patients aged 18 to 85 years who had a discharge diagnosis of myocardial infarction or angina pectoris in 1993-9 and alive in August 1999 were invited by letter; 4373 were randomised.

Interventions Two weeks' treatment with clarithromycin 500 $\mathrm{mg}$ /day or matching placebo.

Main outcome measures Primary outcome: composite of all cause mortality, myocardial infarction, or unstable angina pectoris during three years' follow-up. Secondary outcome: composite of cardiovascular mortality, myocardial infarction, or unstable angina pectoris. The outcomes were obtained from Danish registers and were blindly assessed by the event committee.

Results 2172 participants were randomised to clarithromycin and 2201 to placebo. We found no significant effects of clarithromycin on the primary outcome (hazard ratio 1.15, 95\% confidence interval 0.99 to 1.34 ) or secondary outcome (1.17, 0.98 to 1.40$)$. Mortality was significantly higher in the clarithromycin arm $(1.27,1.03$ to $1.54 ; \mathrm{P}=0.03)$ as a result of significantly higher cardiovascular mortality $(1.45,1.09$ to 1.92 ; $\mathrm{P}=0.01)$.

Conclusions Short term clarithromycin in patients with stable coronary heart disease may cause significantly higher cardiovascular mortality. The long term safety of clarithromycin in patients with stable ischaemic heart disease should be examined.

Trial registration ClinicalTrials.gov NCT00121550.
\end{abstract}

\section{Introduction}

Inflammation may have a fundamental role in coronary heart disease, and infections may promote atherosclerosis or acute coronary syndrome. ${ }^{12}$ Chlamydia pneumoniae has been shown to be present in atherosclerotic tissue. ${ }^{3}$ Macrolide antibiotics are anti-inflammatory and eradicate $C$ pneumoniae from atherosclerotic plaques. ${ }^{45}$ Two small trials showed significant beneficial effects of macrolides on cardiovascular morbidity in patients with acute coronary syndrome ${ }^{6}{ }^{7}$ spurring several randomised trials. Most of these have been meta-analysed, showing that antibiotics do not significantly affect mortality. ${ }^{8}$ The confidence intervals suggest the true effect of antibiotics to lie between an $11 \%$ decrease and a $16 \%$ increase in mortality. ${ }^{8}$ We conducted a large randomised, placebo controlled trial of the effects of clarithromycin on mortality and morbidity of patients with stable coronary heart disease.

\section{Methods}

CLARICOR is a randomised, placebo controlled, multicentre trial in patients with stable coronary heart disease; it has used central randomisation and blinding of all parties in all phases. ${ }^{9}$ The code was not broken until all main analyses were completed and a two way main conclusion formulated.

\section{Organisation}

The Copenhagen Trial Unit coordinated patient identification, mailings, bookings, randomisation, data entry, and data management. Clean data files were exported to the department of biostatistics, University of Copenhagen, for analyses.

\section{Patient recruitment}

All patients discharged from wards or outpatient clinics in the Copenhagen area are registered in a database. We identified all patients with a diagnosis of myocardial infarction or angina pectoris (ICD codes 209-219) during the years 1993-9. We informed patients aged 18 to 85 years who were alive in August 1999 about the trial and invited them to visit one of five cardiology centres staffed by a physician and a nurse. At the visit, we gave patients further information about the trial. We completed an electronic record form with information about previous myocardial infarction, angina pectoris, percutaneous transluminal coronary angioplasty, coronary bypass surgery, arterial hypertension, diabetes mellitus, smoking, and treatment with aspirin, $\beta$ blockers, calcium channel antagonists, long acting nitrates, diuretics, digoxin, statins, and antiarrhythmics. Patients aged 18 to 85 were eligible if they had a history of myocardial infarction, angina, percutaneous transluminal coronary angioplasty, or coronary bypass surgery. Exclusion criteria included myocardial infarction or unstable angina within the previous three months; percutaneous transluminal coronary angioplasty or coronary bypass surgery within the previous six months; New York Heart 
Association class IV cardiac failure; impaired renal or hepatic function; active malignancy; intolerance to macrolides; treatment with methylxanthines, carbamazepine, cisapride, astemizole, terfenadine, or coumarin anticoagulants; participation in other clinical trials within the previous month; incapacity to manage own affairs or sign informed consent; breast feeding; and possible pregnancy.

\section{Randomisation}

A computer generated the allocation sequence and served a telephone voice response randomisation system. Eligible patients were randomly assigned to receive oral clarithromycin $500 \mathrm{mg}$ once daily (Klacid Uno) for two weeks or matching placebo, with stratification by sex, previous myocardial infarction, age below 60 years, and centre in blocks of 10 in a 1:1 ratio. Patients could not be randomised before a complete electronic entry record was saved in the computer. If a patient met exclusion criteria, the program refused randomisation. Blood samples were collected for $C$ pneumoniae serology. ${ }^{9}{ }^{10}$

\section{Follow-up}

All randomised patients received a numbered tablet container and a report form, on which they were asked to mark each tablet taken and to report all adverse events. At the end of treatment, the report form and the tablet container were to be returned to the coordinating centre. If they were not returned, the patient was reminded.

No follow-up visits were planned. Information about death came from the Danish Central Civil Register, which records the vital status of all inhabitants. Information about fatal and non-fatal admissions came from the Danish National Hospital Register, a database of all somatic hospital admissions. Registration is $100 \%$ in these registers. On the basis of these registers, the coordinating centre collected death certificates and copies of hospital records during the follow-up period and forwarded each potential event separately to the event committee. As the observed event rate was lower than estimated, the steering committee extended the follow-up period from two to three years without analysing the data.

\section{Outcome measures}

The primary outcome measure was a composite of all cause mortality, myocardial infarction, or unstable angina. The secondary outcome measure consisted of cardiovascular mortality, myocardial infarction, or unstable angina. The tertiary outcome measure consisted of cardiovascular mortality, myocardial infarction, unstable angina, cerebrovascular attack, or peripheral vascular disease. We analysed mortality as part of a composite outcome $^{11}$ and as a safety measure together with adverse events.

The initial protocol defined the secondary outcome measure as a combination of all cause mortality, myocardial infarction, unstable angina, cerebrovascular attack, or cardiac revascularisation. The steering committee changed this outcome before data analysis in order to focus on cardiac outcomes.

\section{Evaluation of outcome measures}

We used prespecified outcome flowchart forms to search for possible outcomes. We sent copies of hospital records and death certificates to two randomly chosen members of the event committee, which consisted of three cardiologists. In case of disagreement between the two evaluations, we sent both forms together with copies of the record of the event to the third member, who had to select the most likely option. In cases with inadequate information, we classified the cause of death as unknown. If sufficient information was available, we judged death to be due to cardiovascular disease unless a non-cardiovascular cause was present.

An elevation of cardiac enzymes (creatine kinase-isoenzyme MB or troponin) and significant ST changes in the electrocardiograph consistent with myocardial ischaemia or myocardial infarction were required for the diagnoses of myocardial infarction. We classified long lasting chest pain or chest pain at rest without major changes in enzymes as unstable angina. Stroke was focal cerebral deficit lasting more than 24 hours, and transient ischaemic attack was focal cerebral deficit lasting less than 25 hours. Peripheral vascular event encompassed peripheral arterial thromboembolism, surgery or transluminal angioplasty, and severe claudication.

\section{Statistical analyses}

We calculated that at least 2302 patients had to be included in each arm. ${ }^{9}$ The blinded statistical analyses used SAS version $6.12-8$. For time to event variables, we based the hazard ratio and $95 \%$ confidence interval on a Cox regression model. The protocol specified the inclusion of sex, previous myocardial infarction, and age as covariates in the model. We also did multivariate Cox analyses including all entry variables as covariates in turn, supplemented by standard backward selection. We searched for covariate-arm interactions. All analyses were intention to treatthat is, they included all patients as randomised irrespective of compliance. All tests were two sided, and we considered $\mathrm{P}<0.05$ to be significant.

\section{Ethics and safety}

The trial was carried out according to the Helsinki Declaration. We obtained written informed consent before randomisation. The independent data and safety monitoring board did planned interim analyses. If we found conclusive evidence of a benefit of clarithromycin on the primary outcome $(\mathrm{P}<0.001)$ or harm in terms of morbidity and mortality $(\mathrm{P}<0.01)$, the board could recommend early reporting. The Danish Medicines Agency inspected the trial data and the handling of the trial in 2005; the trial had been conducted according to the legislation and protocol.

\section{Results}

Between 5 October 1999 and 15 April 2000, 4373 patients were randomised-2172 to clarithromycin and 2201 to placebo (fig 1)-and started treatment. One placebo patient was HIV positive and was withdrawn at day one. Patients seemed well randomised, although more clarithromycin patients than placebo patients were smokers (table 1).

A total of 4330 patients (clarithromycin 2155/2172, 99.2\%; placebo $2175 / 2200,98.9 \%$ ) returned the record form. A $100 \%$ tablet intake was reported in $90.0 \%$ (1954 patients) in the clarithromycin arm and 93.7\% (2061) in the placebo arm; the $90 \%$ tablet intake was $90.2 \%$ (1959) versus $94.1 \%$ (2070), and the $75 \%$ tablet intake was 91.0\% (1977) versus 94.7\% (2083) (all $\mathrm{P}<0.0001)$. The potential mean follow-up period was 960 days.

\section{Composite outcomes}

The primary outcome (all cause mortality or non-fatal cardiac outcomes) did not differ significantly between the clarithromycin and placebo arms (15.8\% v 13.8\%; hazard ratio $1.15,95 \%$ confidence interval 0.99 to $1.34 ; \mathrm{P}=0.08$ ) (table 2 , fig 2 ).

The secondary outcome (cardiovascular mortality or non-fatal cardiac outcomes) did not differ significantly between the clarithromycin and placebo arms $(11.5 \%$ v 9.9\%; 1.17, 0.98 to $1.40 ; \mathrm{P}=0.09)$ (table 2). 
Table 1 Entry characteristics of patients randomised to clarithromycin or placebo. Values are numbers (percentages) unless stated otherwise

\begin{tabular}{lcc} 
Entry variables & Clarithromycin $(\mathbf{n}=2172)$ & Placebo $(\mathbf{n}=\mathbf{2 2 0 0})$ \\
\hline Mean (SD) age (years) & $65.4(10.3)$ & $65.2(10.4)$ \\
\hline Male & $1514(69.7)$ & $1519(69.1)$ \\
\hline Previous myocardial infarction & $1470(67.7)$ & $1494(67.9)$ \\
\hline Diabetes & $341(15.7)$ & $337(15.3)^{\star}$ \\
\hline Hypertension & $878(40.4)$ & $883(40.2)^{\star}$ \\
\hline Smoking status: & & \\
\hline Smoker & $819(37.7)$ & $753(34.2)$ \\
\hline Ex-smoker & $981(45.2)$ & $1012(46.0)$ \\
\hline Never smoked & $372(17.1)$ & $435(19.8)$ \\
\hline Drugs & & $1937(88.0)$ \\
\hline Aspirin & $1902(87.6)$ & $904(41.1)$ \\
\hline Statin & $896(41.3)$ & $772(35.1)$ \\
\hline Calcium antagonist & $755(34.8)$ & $681(31.0)$ \\
\hline$\beta$ blocker & $653(30.1)$ & $577(26.2)$ \\
\hline ACE inhibitor & $604(27.8)$ & $457(20.8)$ \\
\hline Long acting nitrate & $453(20.9)$ & $126(5.7)$ \\
\hline Digoxin & $154(7.1)$ & $51(2.3)$ \\
\hline Antiarrhythmic & $55(2.5)$ & $762(34.6)$ \\
\hline Diuretic & $773(35.6)$ &
\end{tabular}

ACE=angiotensin converting enzyme inhibitor.

${ }^{*}$ Missing data for one placebo treated patient.

The tertiary outcome measure (cardiovascular mortality, myocardial infarction, unstable angina, cerebrovascular attack, or peripheral vascular disease) was significantly more frequent in the clarithromycin arm than in the placebo arm (16.2\% $v 13.7 \%$; $1.20,1.02$ to $1.39 ; \mathrm{P}=0.03$ ) (table 2 ). The number of non-fatal tertiary outcomes was insignificantly increased by $16 \%$ in the clarithromycin $\operatorname{arm}(1.16,0.97$ to $1.39 ; \mathrm{P}=0.09)$ (table 2).

\section{Mortality}

All cause mortality was significantly higher in the clarithromycin group (1.27, 1.03 to $1.54 ; \mathrm{P}=0.03$ ), as a result of significantly higher cardiovascular mortality $(1.45,1.09$ to $1.92 ; \mathrm{P}=0.01)$ (table 3, fig 3). Non-cardiovascular mortality and non-classified mortality did not differ significantly (table 3 ).

\section{Multivariate analyses}

None of the prespecified outcome measures was significant in Cox regression analyses in which intervention, sex, previous myocardial infarction, age, present smoker status, and ex-smoker
Table 2 Main outcome measures by randomised intervention groups*. Values are numbers (percentages) unless stated otherwise

\begin{tabular}{|c|c|c|c|c|}
\hline & $\begin{array}{c}\text { Clarithromycin } \\
\quad(n=2172)\end{array}$ & $\begin{array}{l}\text { Placebo } \\
(\mathrm{n}=2200)\end{array}$ & $\begin{array}{l}\text { Hazard ratio } \\
(95 \% \mathrm{Cl})\end{array}$ & $P$ value \\
\hline \multicolumn{5}{|l|}{ Primary outcome measure† } \\
\hline All cause mortality & 184 & 159 & & \\
\hline $\begin{array}{l}\text { Myocardial infarction/unstable } \\
\text { angina }\end{array}$ & 160 & 148 & & \\
\hline Total & $344(15.8)$ & $307(13.8)$ & $\begin{array}{c}1.15 \\
\text { (0.99 to } 1.34)\end{array}$ & 0.08 \\
\hline \multicolumn{5}{|l|}{ Secondary outcome measure $†$} \\
\hline Cardiovascular mortality & 89 & 70 & & \\
\hline $\begin{array}{l}\text { Myocardial infarction/unstable } \\
\text { angina }\end{array}$ & 160 & 148 & & \\
\hline Total & $249(11.5)$ & $218(9.9)$ & $\begin{array}{c}1.17 \\
(0.98 \text { to } 1.40)\end{array}$ & 0.09 \\
\hline \multicolumn{5}{|l|}{ Tertiary outcome measure $†$} \\
\hline Cardiovascular mortality & 83 & 65 & & \\
\hline $\begin{array}{l}\text { Myocardial infarction/unstable } \\
\text { angina }\end{array}$ & 153 & 144 & & \\
\hline Stroke & 81 & 68 & & \\
\hline Peripheral vascular disease & 34 & 26 & & \\
\hline Total & $351(16.2)$ & $303(13.7)$ & $\begin{array}{c}1.20 \\
(1.02 \text { to } 1.39)\end{array}$ & 0.03 \\
\hline
\end{tabular}

*Based on a Cox regression model including sex, previous myocardial infarction, and age as mandatory covariates.

†Patients censored after the first event.

status were fixed covariates and other covariates were included as described in the methods. The non-significant tendency towards increased risk in the clarithromycin group persisted in the multivariate analyses of the primary outcome $(1.11,0.95$ to 1.30 ; $\mathrm{P}=0.17)$, the secondary outcome $(1.14,0.95$ to $1.37 ; \mathrm{P}=0.15)$, and the tertiary outcome $(1.16,1.00$ to $1.35 ; \mathrm{P}=0.06)$. All cause mortality was insignificantly increased (1.21, 0.99 to 1.48; $\mathrm{P}=0.07$ ), and cardiovascular mortality continued to be significantly increased in the clarithromycin group $(1.38,1.03$ to $1.85 ; \mathrm{P}=0.03)$.

\section{Chlamydia pneumoniae antibodies}

An entry blood sample was available for $4350 / 4372(99.5 \%)$ patients. We found a $C$ pneumoniae IgG antibody titre of 64 or above in 1390/2162 (64.3\%) patients in the clarithromycin arm and $1377 / 2188(62.9 \%)$ in the placebo arm. The corresponding figures for IgA antibody titres of 64 or above were $488 / 2162$

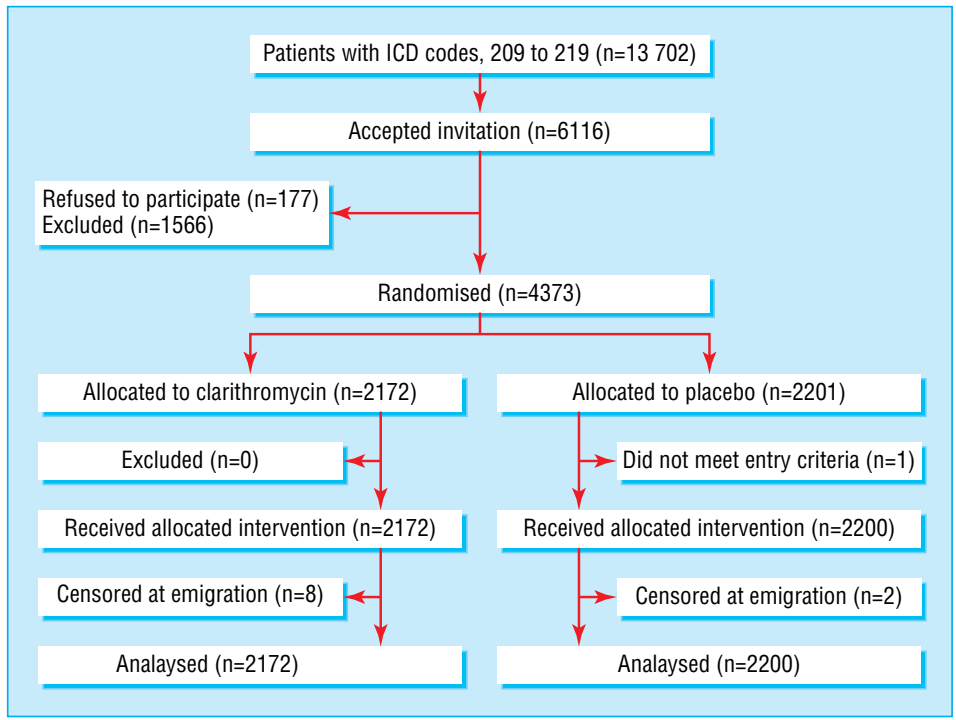

Fig 1 Flow of participants through enrolment, randomisation, and follow-up of CLARICOR trial 


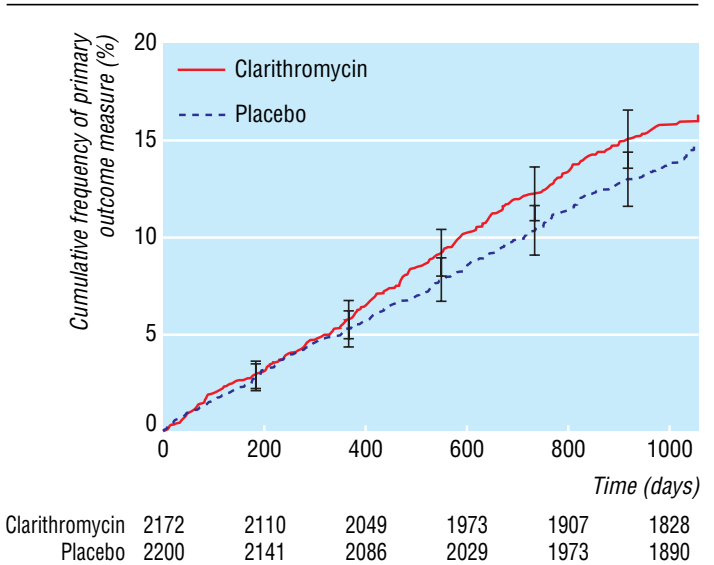

Fig 2 Kaplan-Meier estimate (with 95\% confidence intervals) of the primary outcome measure (composite of all cause mortality, myocardial infarction, or unstable angina pectoris) according to intervention. Figures below the diagram are biannual numbers at risk in the clarithromycin and placebo groups

(22.6\%) and 469/2188 (21.4\%). The serological data did not co-vary significantly with arm, outcomes, or drug effect (data not shown).

\section{Adverse events}

During treatment, 851 (39.5\%) clarithromycin patients and 547 (25.1\%) placebo patients reported at least one adverse event $(\mathrm{P}=0.0001)$. One serious adverse event (acute nephropathy) led to unmasking (placebo). During the first month, 19 clarithromycin patients and 13 placebo patients were admitted to hospital with abdominal discomfort and two clarithromycin and four placebo patients died from cardiovascular causes. These differences were not significant.

Table 3 Mortality according to randomised intervention groups*. Values are numbers (percentages) unless stated otherwise

\begin{tabular}{lcccc} 
Mortality & $\begin{array}{c}\text { Clarithromycin } \\
(\mathbf{n}=\mathbf{2 1 7 2})\end{array}$ & $\begin{array}{l}\text { Placebo } \\
(\mathbf{n}=2200)\end{array}$ & $\begin{array}{c}\text { Hazard ratio }(\mathbf{9 5 \%} \\
\mathbf{C I})\end{array}$ & P value \\
\hline Cardiovascular & $111(5.1)$ & $78(3.5)$ & $1.45(1.09$ to 1.92$)$ & 0.01 \\
\hline Non-cardiovascular & $85(3.9)$ & $84(3.8)$ & $1.03(0.76$ to 1.41$)$ & 0.82 \\
\hline Non-classified & $16(0.7)$ & $10(0.5)$ & $1.64(0.75$ to 2.11$)$ & 0.22 \\
\hline All cause & $212(9.8)$ & $172(7.8)$ & $1.27(1.03$ to 1.54$)$ & 0.03 \\
\hline
\end{tabular}

*Based on Cox regression model including sex, previous myocardial infarction, and age as mandatory covariates. All non-fatal events were ignored.

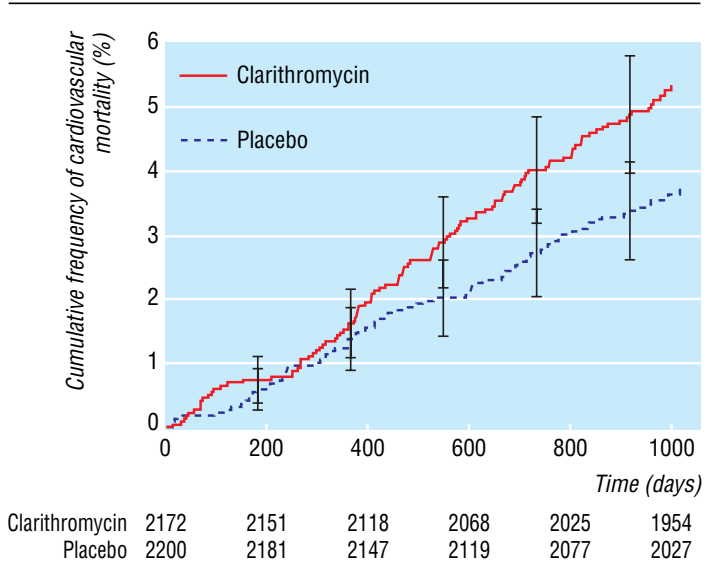

Fig 3 Kaplan-Meier estimate (with 95\% confidence intervals) of cardiovascular mortality according to intervention. Figures below the diagram are biannual number at risk in the clarithromycin and placebo groups

\section{Discussion}

We found no beneficial effect of short term clarithromycin for patients with stable coronary heart disease. On the contrary, the significantly increased cardiovascular mortality in the clarithromycin treated patients surprised us.

\section{Strengths and limitations}

The major strengths were the size of the trial, the central randomisation, stratification at randomisation, and the placebo controlled intervention with blinded outcome assessment and intention to treat analyses. Compliance was excellent. We obtained all outcomes from public registers, securing follow-up of more than $99 \%$ of patients. The event committee followed stable criteria uniformly and blindly classified events. These safeguards protected against random errors and systematic errors (bias). ${ }^{12-14}$

Potential weaknesses include the fact that only $32 \%$ of potentially eligible patients were randomised, which may affect the external validity. Furthermore, we cannot exclude chance imbalance at randomisation or after randomisation as a cause of the increased mortality. We would like to know the New York Heart Association class and ejection fraction at randomisation and medical treatment and lifestyle during follow-up. We find it unlikely that these factors would differ substantially between the two groups, which seemed well randomised, but we cannot exclude chance imbalances. Clarithromycin patients were more often smokers. However, our multivariate analyses taking smoking and other entry variables into consideration did not substantially change the results.

\section{Clarithromycin effects}

Why clarithromycin might have an unfavourable effect in patients with coronary heart disease remains unclear. Macrolides possess-apart from their antibiotic characteristics-potassium channel blocker properties, ${ }^{15}$ which can cause prolonged QT intervals, torsades de pointes tachycardia, and death. This risk is significantly increased by coadministration of macrolides and drugs metabolised by cytochrome $\mathrm{P} 4503 \mathrm{~A}$ isoenzymes. ${ }^{16}$ We did not observe differences in cardiovascular mortality during the first month, and drug interactions cannot readily explain the observed difference in cardiovascular mortality.

\section{Comparison with related research}

Only two other trials have assessed clarithromycin in patients with coronary heart disease. ${ }^{17}{ }^{18}$ The CLARIFY trial randomised 148 patients to clarithromycin or placebo for three months. The clarithromycin group had an insignificantly raised 1.5 year mortality (odds ratio $4.17,95 \%$ confidence interval 0.46 to 38.2 ). ${ }^{17}$ Berg et al randomised 473 patients to clarithromycin or placebo until the day of coronary artery bypass grafting. ${ }^{18}$ Two year mortality was insignificantly raised in the clarithromycin patients $(1.10,0.44$ to 2.76$){ }^{18}$ Pooling these data with our results shows a significantly increased mortality in clarithromycin patients (1.28, 1.05 to 1.57$)$.

Several trials on azithromycin, roxithromycin, or gatifloxacin in patients with coronary heart disease have been published. ${ }^{8}{ }^{19-}$ ${ }_{25}$ The overall conclusion is that antibiotics have no significant impact on cardiovascular events. ${ }^{84}{ }^{25}$ Two of the trials reported a short lived beneficial effect. ${ }^{21}{ }^{22}$ We found a gradually increasing outcome rate in the clarithromycin patients (fig 2 and fig 3 ). If we had stopped follow-up at one year, we would have had a neutral result like most other trials. ${ }^{8}$ If we pool the data from the Andraws et al meta-analysis, ${ }^{8}$ the Berg et al trial, ${ }^{18}$ and our trial, antibiotics irrespective of type and duration of follow-up do not 


\section{What is already known on this topic}

Studies have shown an association between Chlamydia pneumoniae serological markers and cardiovascular events

Randomised trials (mostly under two years in length) have shown variable effects of antibiotics in patients with acute or stable coronary heart disease

A meta-analysis found no significant effects of antibiotics in coronary patients, but the $95 \%$ confidence interval indicates an effect between an $11 \%$ decrease and a $16 \%$ increase in mortality

\section{What this study adds}

Clarithromycin $500 \mathrm{mg}$ /day for 14 days compared with placebo seemed to cause more adverse effects during tablet intake in patients with stable coronary heart disease

Clarithromycin may cause increased mortality (hazard ratio $1.27,95 \%$ confidence interval 1.03 to 1.54 ) and increased cardiovascular mortality $(1.45,1.09$ to 1.92$)$ in patients with stable coronary heart disease followed for up to three years

significantly affect mortality $(1.09,0.97$ to 1.22$)$. If we pool the data of the three trials following patients for more than two years (PROVE-IT, ${ }^{19}$ ACES, ${ }^{23}$ and CLARICOR), antibiotics are associated with significantly increased mortality $(1.20,1.04$ to 1.39$)$.

\section{Conclusions}

Brief intervention with clarithromycin in patients with stable coronary heart disease living in Copenhagen may cause more cardiovascular deaths. The long term safety of clarithromycin in patients with coronary heart disease needs further examination. We therefore recommend other researchers to assess the long term impact of clarithromycin and other antibiotics. At present, no evidence indicates that antibiotics have protective effects in patients with atherosclerotic cardiovascular disease. Other roads should properly be taken. ${ }^{25}$

We thank Jørn Wetterslev for help during the inspection of the CLARICOR trial.

Contributors: HJK had the original idea for the trial. CMJ chaired the steering committee, which consisted of JFH, PH, GBJ, JK, HJK, IL, HN, and CG. $\mathrm{CMJ}$, JFH, PH, GBJ, JK, IL, HN, and CG were principal investigators. BA-N, $\mathrm{MD}$, LP, SH, and OHH were clinical investigators. CMJ and CG were coordinating investigators and drafted the protocol and this report. CG is the guarantor. EK was chair of the event committee. JH was a member of the independent data monitoring and safety committee and did the statistical analyses. Of the contributors who are not listed as authors, Jette Mieritz (Amager Hospital); Lene Thuesen (Bispebjerg Hospital); Hanne Larsen, Per Nielsen, and Merethe Hildebrandt (Frederiksberg Hospital); Mette Bommersholdt (Hvidovre Hospital); and Lone Kristensen (Rigshospitalet) were study nurses. Jette V Petersen and Pia Hughes coordinated the trial data accrual and data entry. Bitten Hansen, Ninna Frydendahl, Bessie Hødholdt, Karen Juliussen, and Mette Hansen executed data accrual. Kjell Nillson and Nader Salas developed the randomisation and data management systems. Nader Salas was responsible for data management. Christina Nørgaard and Lene Berthelsen, of the Neisseria Unit, Statens Serum Institut, Copenhagen, Denmark, did the serological tests. EK, Niels Frandzen, and Søren Gallatius constituted the event committee and assessed all outcomes. The independent data monitoring and safety committee was chaired by Kristian Thygesen (Århus, Denmark) with John Kjekshus (Oslo, Norway) and JH (Copenhagen) as members. Stig Haunsø (Rigshospitalet, Copenhagen), Rolf Steffensen (KAS Gentofte, Hellerup, Denmark), and Thorkild IA Sørensen (Institute of Preventive Medicine, Copenhagen) advised the steering group during the planning of the trial.

Funding: The CLARICOR trial is investigator initiated and controlled. This work was supported by grants from non-profit funds (Danish Heart Foun- dation, Copenhagen Hospital Corporation, Danish Research Council, 1991 Pharmacy Foundation). Abbott Laboratories, IDC, Queensborough, UK, supplied the clarithromycin and placebo tablets. The organisations supporting the trial had no role in design, data collection, data analyses, data interpretation, or writing the report. The steering group had full access to all the data and had final responsibility for the decision to submit the report for publication.

Competing interests: None declared.

Ethical approval: The trial was approved by the local ethics committee (KF 01-076/99), the Danish Medicines Agency (2612-975), and the Danish Data Protection Agency (1999-1200-174).

1 Libby P, Ridker PM, Maser A. Inflammation and atherosclerosis. Circulation 2002;105:1135-43.

2 Muhlestein JB, Anderson JL. Infectious serology and atherosclerosis: how burdensome is the risk? Circulation 2003;107:220-2

3 Gelfand EV, Cannon CP. Antibiotics for secondary prevention of coronary artery disease: an ACES hypothesis but we need to PROVE IT. Am Heart J 2004;147:202-9.

4 Gieffers J, Solbach W, Maass M. In vitro susceptibility and eradication of Chlamydia pneumoniae cardiovascular strains from coronary artery endothelium and smooth muscle cells. Cardiovasc Drugs Ther 2001;15:259-62.

5 Melissano G, Blasi F, Esposito G, Tarsia P, Dordoni L, Arosio C, et al. Chlamydia pneumoniae eradication from carotid plaques: results of an open, randomised treatment study. Eur I Vasc Endovasc Surg 1999;18:355-9.

6 Gupta S, Leatham EW, Carrington D, Mendall MA, Kaski JC, Camm AJ. Elevated C. pneumoniae antibodies, cardiovascular events, and azithromycin in male survivors of pneumoniae antibodies, cardio
MI. Circulation 1997;96:404-7.

7 Gurfinkel E, Bozovich G, Daroca A, Beck E, Mautner B. Randomised trial of roxithromycin in non-Q-wave coronary syndromes: ROXIS pilot study. Lancet 1997;350:404-7.

8 Andraws R, Berger JS, Brown DL. Effects of antibiotic therapy on outcomes of patients with coronary artery disease: a meta-analysis of randomized controlled trials. JAMA 2005;293:2641-7.

9 Hansen S, Als-Nielsen B, Damgaard M, Helø OH, Petersen L, Jespersen CM, et al. Intervention with clarithromycin in patients with stable coronary heart disease: the CLARICOR trial design. HeartDrug 2001;1:14-9.

10 Bennedsen M, Berthelsen L, Lind I, the IAMA Group. Performance of three microimmunofluorescence assays for detection of Chlamydia pneumoniae immunoglobulin M, Gunofluorescence assays for detection of Chlamydia pneur

11 Freemantle N, Calvert M, Wood J, Eastaugh J, Griffin C. Composite outcomes in randomized trials: greater precision but with greater uncertainty? JAMA 2003;289:2554-9.

12 Schulz KF, Chalmers I, Hayes RJ, Altman DG. Empirical evidence of bias: dimensions of methodological quality associated with estimates of treatment effects in controlled trials. JAMA 1995;273:408-12.

13 Moher D, Pham B, Jones A, Cook DJ, Jadad AR, Moher M, et al. Does quality of reports of randomised trials affect estimates of intervention efficacy reported in meta-analyses? Lancet 1998;352:609-13.

14 Kjærgard LL, Villumsen J, Gluud C. Reported methodological quality and discrepancies between large and small randomized trials in meta-analyses. Ann Intern Med 2001;135:982-9.

15 Martin D, Bursill J, Qui MR, Breit SN, Campbell T. Alternative hypothesis for efficacy of macrolides in acute coronary syndromes. Lancet 1998;351:1858-9.

16 Ray WA, Murray KT, Meredith S, Narasimhulu SS, Hall K, Stein M. Oral erythromycin and the risk of sudden death from cardiac causes. N Engl J Med 2004;351:1089-96.

17 Sinisalo J, Mattila K, Valtonen V, Anttonen O, Juvonen J, Melin J, et al. Effect of 3 months of antimicrobial treatment with clarithromycin in acute non- $Q$-wave coronary syndrome. Circulation 2002;105:1555-60.

18 Berg HF, Maraha B, Scheffer G-J, Quarles-van Ufford M, Vandenbroucke-Grauls CM, Peeters MF, et al. Treatment with clarithromycin prior to coronary artery bypass graft surgery does not prevent subsequent cardiac events. Clin Infect Dis 2005;40:358-65.

19 Cannon CP, Braunwald E, McCabe CH, Grayston JT, Muhlestein B, Giugliano RP, et al. Antibiotic treatment of Chlamydia pneumoniae after acute coronary syndrome. N Engl IMed 2005;352:1646-54.

20 Cercek B, Shah PK, Noc M, Zahger D, Zeymer U, Matetzky S, et al. Effect of short-term treatment with azithromycin on recurrent ischaemic events in patients with acute coronary syndrome in the azithromycin in acute coronary syndrome (AZACS) trial: a randomised controlled trial. Lancet 2003;361:809-13.

21 Gurfinkel E, Bozovich G, Beck E, Testa E, Livellara B, Mautner B. Treatment with the antibiotic roxithromycin in patients with acute non-Q-wave coronary syndromes: the final report on the ROXIS study. Eur Heart J 1999;20:121-7.

22 O'Connor CM, Dunne MW, Pfeffer MA, Muhlestein JB, Yao L, Gupta S, et al. Azithromycin for the secondary prevention of coronary heart disease events: the WIZARD study: a randomized controlled trial. JAMA 2003;290:1459-66.

23 Grayston JT, Kronmal RA, Jackson LA, Parisi AF, Muhlestein JB, Cohen JD, et al. Azithromycin for the secondary prevention of coronary events. $N$ Engl J Med 2005;352:1637-45.

24 Danesh J. Antibiotics in the prevention of heart attacks. Lancet 2005;365:365-6.

24 Danesh J. Antibiotics in the prevention of heart attacks. Lancet 2005;365:365-6.
25 Taylor-Robinson D, Boman J. The failure of antibiotics to prevent heart attacks: it's not Taylor-Robinson D, Boman J. The failure of antibiotics
necessarily the end of the road. BMJ 2005;331:361-2.

(Accepted 17 October 2005)

doi $10.1136 /$ bmj.38666.653600.55

Bispebjerg Hospital, Copenhagen University Hospital, Department of Cardiology Y, Bispebjerg Bakke 23, DK-2400 Copenhagen, Denmark

Christian M Jespersen consultant physician in cardiology

Morten Damgaard registrar

Jørgen Fischer Hansen head of department

Amager Hospital, Copenhagen University Hospital, Department of Cardiology, 
DK-2300 Copenhagen

Bodil Als-Nielsen registrar

Henrik Nielsen head of department

Hvidovre Hospital, Copenhagen University Hospital, Department of Cardiology,

DK-2650 Hvidovre, Denmark

Stig Hansen registrar

Gorm B Jensen head of department

Rigshospitalet, Copenhagen University Hospital, The Heart Centre, Department of Medicine B, DK-2100 Copenhagen

Olav H Helø registrar

Jens Kastrup head of department

Frederiksberg Hospital, Copenhagen University Hospital, Department of

Cardiology E, DK-2000 Frederiksberg, Denmark

Lars Petersen registrar

Per Hildebrandt head of department

Department of Biostatistics, Institute of Public Health, Faculty of Health Sciences,
University of Copenhagen, DK-2200 Copenhagen

Jørgen Hilden associate professor of statistics

Department of Clinical Microbiology, Odense University Hospital, DK-5000 Odense, Denmark

Hans Jørn Kolmos professor of microbiology

Statens Serum Institut, DK-2300 Copenhagen

Inga Lind consultant physician

Herlev Hospital, Copenhagen University Hospital, Department of Cardiology S, DK-2730 Herlev, Denmark

Erik Kjøller consultant physician in cardiology

The Copenhagen Trial Unit, Centre for Clinical Intervention Research, Institute of Preventive Medicine and Rigshospitalet, Copenhagen University Hospital, Blegdamsvej 9, DK-2100 Copenhagen

Christian M Jespersen consultant physician in cardiology

Christian Gluud head of department

Correspondence to: C M Jespersen cmj01@bbh.hosp.dk 